\title{
Propagation model for the dynamics of gain-guided semiconductor laser arrays
}

\author{
Shawe-Shiuan Wang and Herbert G. Winful \\ Department of Electrical Engineering and Computer Science, University of Michigan, Ann Arbor, \\ Michigan 48109-2122
}

(Received 16 June 1992; accepted for publication 22 September 1992)

\begin{abstract}
A model is presented for the spatiotemporal dynamics of gain-guided semiconductor laser arrays. The model goes beyond coupled mode theory and treats the array as a single entity. Numerical simulations of twin-stripe gain-guided arrays yield stable or pulsing outputs, depending on array parameters.
\end{abstract}

Semiconductor laser arrays are becoming attractive sources for applications that require high power in a wellcollimated beam. ${ }^{1}$ To identify optimal structures for laser arrays, it is necessary to understand the dynamics of these devices. In recent articles we have used coupled mode theory to describe the dynamic behavior of laser arrays and have discovered coupling-induced instabilities in these lasers. ${ }^{2,3}$ Coupled mode theory, however, is only strictly valid for structures such as weakly coupled index-guided arrays where the modes of the individual lasers are well confined and thus serve as proper basis fields for the coupled mode description. In this article we present a dynamic propagation model that goes beyond coupled mode theory and treats the array as a single entity. We apply the model to twin-stripe gain-guided laser arrays and find stable or unstable outputs, depending on the spacing between the emitters.

The model is based on the approach used by Agrawal to describe the steady-state behavior of the laser array lateral modes. ${ }^{4}$ By extending that work to include the dynamics of the charge carriers, we obtain a self-consistent description of the temporal evolution of the light field in the array.

The geometry of the laser array is shown in Fig. 1. The electric field of the light wave is taken as

$$
\bar{E} \simeq \hat{x} \operatorname{Re}\{\Psi(x, z ; t) \phi(y) \exp [i(k z-\omega t)]\}
$$

where $\phi(y)$ is the transverse field distribution of the fundamental transverse electric (TE) mode, and $\Psi(x, z ; t)$ is the lateral field distribution whose amplitude may depend on time. Within the effective index approximation, the propagation of the lateral field $\Psi$ in the laser cavity is described by the paraxial wave equation ${ }^{4}$

$$
2 i k \frac{\partial \Psi}{\partial z}+\frac{\partial^{2} \Psi}{\partial x^{2}}+k_{0}^{2} \Gamma \Delta \epsilon \Psi=0
$$

where $\Gamma$ is the active layer confinement factor, $k$ is the propagation constant, $k_{0}$ is its value in free space, $x$ is the lateral direction, and $z$ is the direction of propagation. The dielectric perturbation $\Delta \epsilon$ represents the distribution of gain, loss, and refractive index in the lateral direction. It depends on the carrier density $N$ through ${ }^{4}$

$$
\begin{aligned}
\Delta \epsilon= & -a \eta_{a} \alpha N / k_{0}-i\left(\eta_{a} / k_{0}\right) a\left(N-N_{0}\right) \\
& +i\left(\eta_{c} / k_{0}\right)(1-\Gamma) \alpha_{c} / \Gamma,
\end{aligned}
$$

where $\eta_{a}$ and $\eta_{c}$ are the refractive indices of the active layer and cladding layers, $a$ is the gain coefficient, $\alpha_{c}$ is the loss in the cladding-layer, $\alpha$ is the linewidth enhancement factor (also known as the antiguiding parameter), and $N_{0}$ is the carrier density required to achieve transparency. The carrier density $N(x, t)$ satisfies the diffusion equation ${ }^{5}$

$$
\frac{\partial N(x)}{\partial t}=P(x)-\frac{N(x)}{\tau_{s}}-\Gamma g^{\prime} N(x) I(x)+D_{x x} \frac{\partial^{2} N(x)}{\partial x^{2}} .
$$

Here, $P$ is the pump rate, $g^{\prime}$ is the differential gain, $\tau_{s}$ is the carrier lifetime, $I(x)=|\Psi(x)|^{2}$ is the average light distribution in one round trip, and $D_{x x}$ is the carrier diffusion coefficient.

In solving Eqs. (2)-(4), we make a number of simplifying assumptions to save on computer time. We neglect variations in the carrier density in the longitudinal direction as well as interference effects between forward and backward waves in the cavity. Starting with an initial arbitrary field distribution at the front facet and a longitudinally uniform carrier density, the beam propagation method is used with Eq. (2) to propagate this field to the rear facet. The field is attenuated upon reflection and the propagated back to the front facet. After each round trip the carrier density is updated by solving Eq. (4) using the Crank-Nicolson method. ${ }^{6}$ Our basic time step is thus the cavity round trip time, which in these simulations is about $5 \mathrm{ps}$. The field amplitude, the total intensity in each stripe, and the phase distribution in the lateral direction can be recorded at the end of each round trip of the wave in the laser cavity. Furthermore, the far-field intensity distribution can be calculated by taking the Fourier transform of the near-field amplitude and phase distribution.

An equivalent approach, which is more in the spirit of the "mean-field" theory of nonlinear resonators, ${ }^{7}$ is to consider $\Psi$ as the amplitude of a single longitudinal mode in the cavity. The mirror losses are then assumed to be distributed throughout the cavity and added to Eq. (3) for the dielectric perturbation. The derivative with respect to $z$ in Eq. (2) is replaced by a temporal derivative and we obtain 


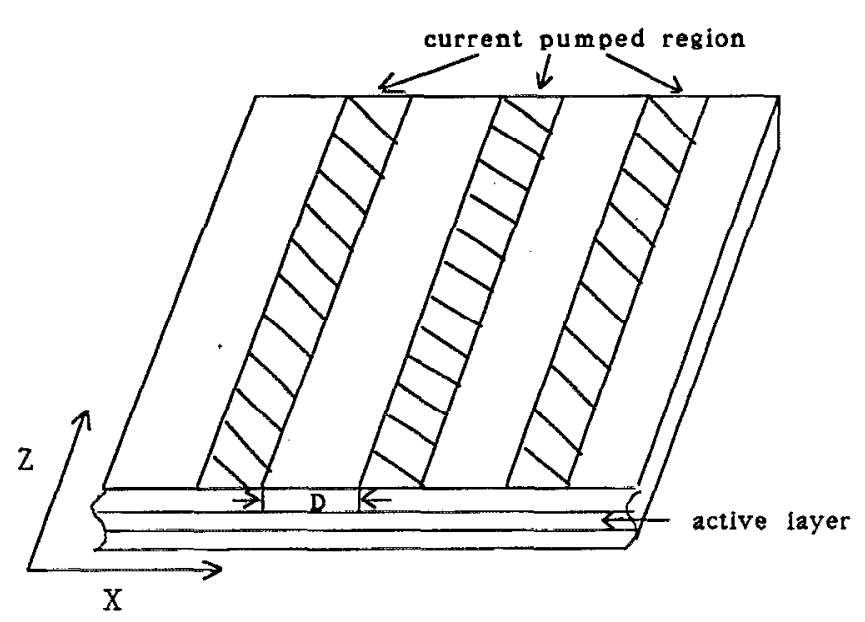

FIG. 1. Geometry of the gain-guided laser array.

$$
\frac{2 i k}{c} \frac{\partial \Psi}{\partial t}+\frac{\partial^{2} \Psi}{\partial x^{2}}+k_{0}^{2} \Gamma \Delta \epsilon \Psi=0 .
$$

Equation (5) with distributed loss yields the same numerical results as Eq. (2).

We have used this model to simulate the behavior of twin-stripe gain guided lasers. For the simulations reported here, the width of each stripe is $5 \mu \mathrm{m}$ and the length of the laser cavity is $250 \mu \mathrm{m}$. In solving Eq. (2) by the beam propagation method, the lateral $(x)$ dimension is discretized into 1024 elements while the longitudinal $(z)$ dimension is divided into 500 steps. The pump current distribution is uniform underneath the pumping stripe and decays exponentially in the unpumped regions. The pump rate is taken as

$P(x)= \begin{cases}P_{0}=1.83 \times 10^{15} \mathrm{~s}^{-1} \mu \mathrm{m}^{-3} & \text { if }\left|x-x_{0}\right|<2 \mu \mathrm{m}, \\ P_{0} \exp \left[-\left(\frac{\left|x-x_{0}\right|-2}{l_{0}}\right)\right] & \text { otherwise. }\end{cases}$

Here, $l_{0}=2 \mu \mathrm{m}$ is the current spreading factor and $x_{0}$ locates the center of the stripe. The facet reflectivity is equal to 0.36 . The values of the other parameters are $N_{0}=1.0$ $\times 10^{6} \mu \mathrm{m}^{-3}, \lambda=0.87 \mu \mathrm{m}, D_{x x}=3.3 \mu \mathrm{m}^{2} / \mathrm{ns}, \eta_{a}=3.59, \alpha_{c}$ $=0.001 \mu \mathrm{m}^{-1}, \tau_{s}=2 \mathrm{~ns}, \tau_{p}=1 \mathrm{ps}$, and $\alpha=3.0$. The relation between $g^{\prime}$ and $a$ is $\Gamma g^{\prime}=\Gamma a v_{g}=0.8 \times 10^{6} \mu \mathrm{m}^{3} / \mathrm{s}$, where $v_{g}=0.833 \times 10^{14} \mu \mathrm{m} / \mathrm{s}$ is the group velocity.

Previous coupled mode analysis of laser array dynamics has shown that the stability of these lasers depends critically on the strength of the coupling between them. ${ }^{3}$ For the propagation model presented here, we vary the strength of coupling between the two stripes by changing the width $D$ of the central unpumped region. Figure 2 shows the temporal evolution of light intensity from one stripe for $D=36 \mu \mathrm{m}$. Because the central absorption region is so wide, light from one stripe has only a slight effect on the other and the two lasers are almost isolated from each other. The output therefore exhibits damped relaxation oscillations and reaches a stable steady state as would be expected for uncoupled, narrow-stripe gain-guided lasers.

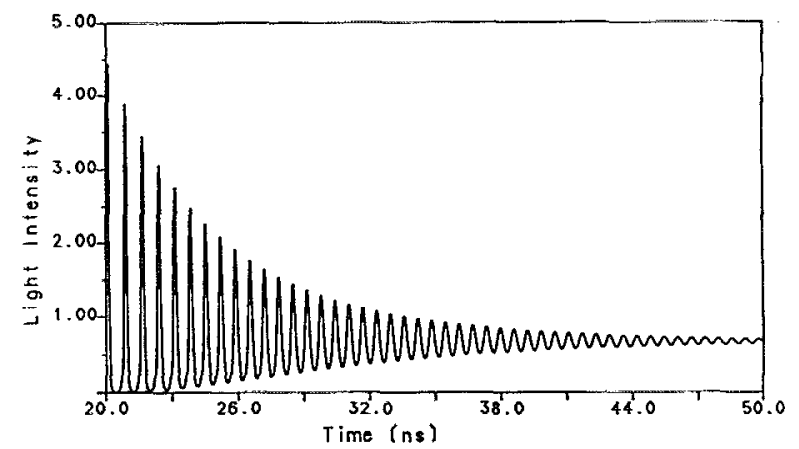

FIG. 2. Temporal evolution of the light intensity in one stripe of the twin stripe laser. Here the spacing between the stripes is $D=36.0 \mu \mathrm{m}$.

At steady state, there is a $\pi$ phase difference between the fields in the two stripes.

Figure 3(a) shows the output from one stripe when the width of the absorption region is reduced to $16 \mu \mathrm{m}$. With the increased coupling between the lasers, the output exhibits sustained pulsations that have the character of $Q$ switched spikes. The time interval between the spikes is about $2.0 \mathrm{~ns}$, of the order of the carrier lifetime. The pulse width (FWHM) is about 40 ps and the modulation depth is close to $100 \%$. Figure $3(\mathrm{~b})$ shows the spatio-temporal evolution of the output intensity in the self- $Q$-switched mode. It is seen that the entire lateral mode profile pulsates in synchronism and that there is no exchange of energy between the two stripes. Similar synchronous pulsations have been observed in experiments on twin-stripe gainguided laser arrays. ${ }^{8}$ It should be noted that the shape of the lateral mode remains invariant throughout the self pulsations. Furthermore, a fixed phase difference of $\pi$ is maintained between the complex fields in the two stripes. This means that the field in this two-stripe device is locked into an out-of-phase lateral mode. The far-field pattern associated with the self-Q-switched mode is shown in Fig. 3(c). The absence of a central lobe (at $\theta=0^{\circ}$ ) is a consequence of the $\pi$ phase difference between the fields in the two stripes.

The mechanism of the sustained pulsations seen here is similar that of other multisection diode lasers. ${ }^{9,10}$ The unpumped (or, rather, weakly pumped) region between the lasers acts as a saturable absorber and leads to repetitive $Q$ switching. Other recent authors have observed pulsing behavior in their numerical simulation of these lasers and have attributed the phenomenon to transverse mode competition. ${ }^{12}$ In our case such a description does not apply since the output remains in a fixed lateral mode throughout the pulsations.

The simulations presented here do not include the effect of nonlinear gain or of spontaneous emission into the lasing mode. Both phenomena introduce extra damping into the system and will reduce the amplitude of the selfpulsations. In future work we will address the impact of these neglected terms on the stability domain of coupled lasers.

In summary, this study has successfully simulated 

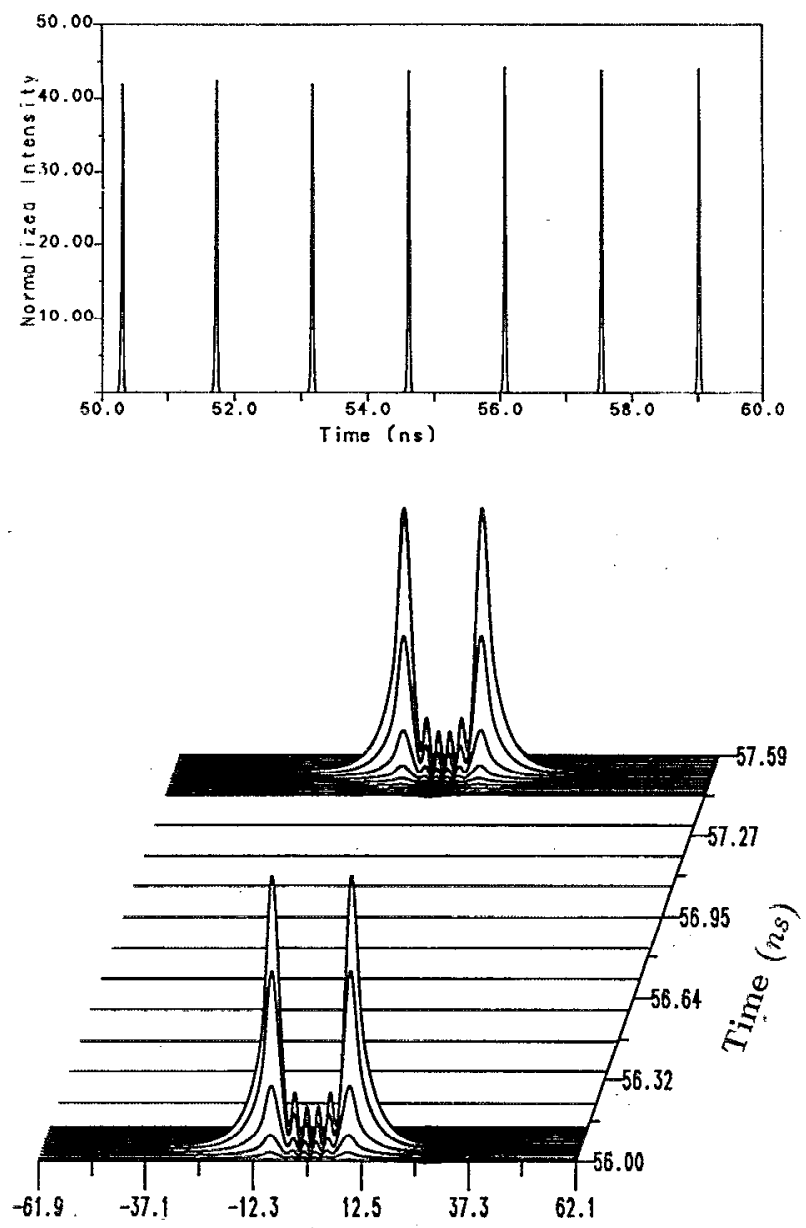

Lateral Dimension $(\mu m)$

gain-guided twin-stripe semiconductor lasers via a propagation model. Both stable and unstable (pulsing) results are obtained, depending on array parameters. In part of the unstable region, we observe repetitive $Q$ switching in a fixed lateral mode.

Partial support for this research is provided by the National Science Foundation through grants ECS-8906214 and ECS-8657256. Additional funding was provided by the U.S. Air Force Office of Scientific Research.

${ }^{1}$ For a recent review, see D. Botez and D. E. Ackley, IEEE Circuits Devices Mag. 2, 8 (1986).

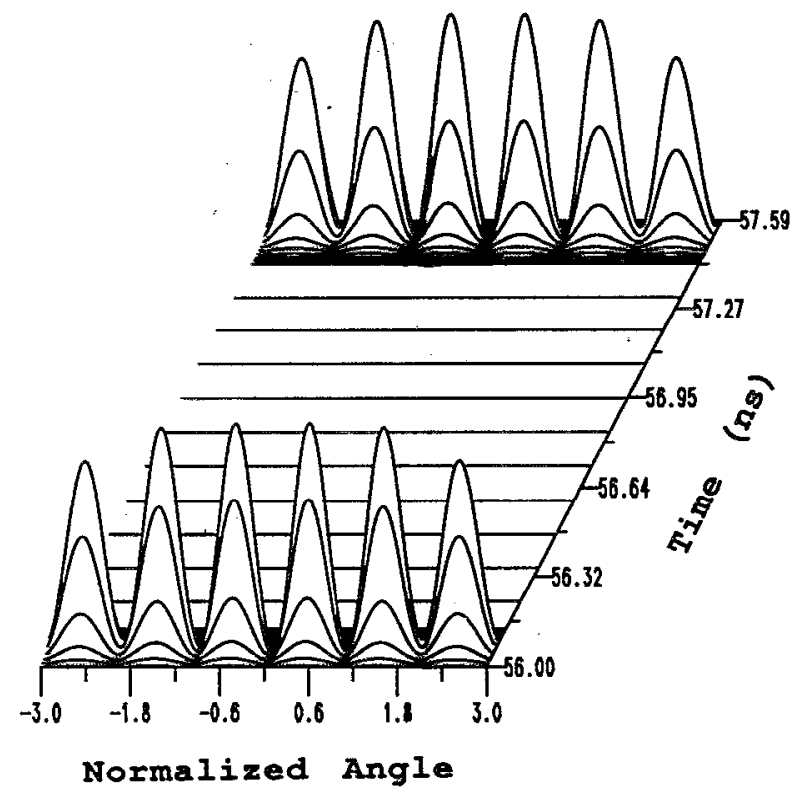

FIG. 3. (a) Temporal evolution of the light intensity in the near field of a twin stripe laser under self-pulsing conditions. Here the spacing between the stripes is $D=16.0 \mu \mathrm{m}$. (b) Spatio-temporal evolution of the near-field intensity under self-pulsing conditions. (c) Spatio-temporal evolution of the far-field intensity corresponding to the near field of (b). The normalized angle is $\delta=(s / \lambda) \sin (\theta)$, where $S$ is the center-to-center distance between the two stripes, $\lambda$ is the wavelength in the free space, and $\theta$ is the diffraction angle.

${ }^{2}$ S. S. Wang and H. G. Winful, Appl. Phys. Lett. 52, 1774 (1988).

${ }^{3}$ H. G. Winful and S. S. Wang, Appl. Phys. Lett. 53, 1894 (1988).

${ }^{4}$ G. P. Agrawal, J. Appl. Phys. 58, 2922 (1985).

${ }^{5}$ G. R. Hadley, J. P. Hohimer, and A. Owyoung, IEEE J. Quantum Electron. QE-24, 2138 (1988).

${ }^{6}$ R. L. Burden and J. D. Faires, Numerical Analysis (PWS, Boston, 1985).

${ }^{7}$ L. A. Lugiato and R. Lefever, Phys. Rev. Lett. 58, 2209 (1987).

${ }^{8}$ R. K. DeFreez and N. Yu (personal communication).

${ }^{9}$ I. S. Goldobin, V. N. Luk'yanov, A. F. Solodkov, and S. D. Vakubovich, Soy. J. Quantum Electron. 15, 627 (1985), and references therein.

${ }^{10}$ T. E. Rozzi and K. A. Shore, J. Opt. Soc. Am. B 2, 237 (1985).

${ }^{11}$ P. Menendez-Valdes, E. Garmire, and M. Ohtaka; IEEE J. Quantum Electron. QE-26, 2075 (1990). 